\title{
Grain Boundary Structure Dependence of Extrinsic Grain Boundary Dislocation Emission Phenomena: A Molecular Dynamics Study
}

\author{
Keisuke Kinoshita ${ }^{1, *}$, Tomotsugu Shimokawa ${ }^{2}$ and Toshiyasu Kinari ${ }^{2}$ \\ ${ }^{1}$ Graduate School of Natural Science and Technology, Kanazawa University, Kanazawa 920-1192, Japan \\ ${ }^{2}$ College of Science and Engineering, Kanazawa University, Kanazawa 920-1192, Japan
}

Recently, it has been reported that extrinsic grain boundary dislocations (EGBDs) are often present in the grain boundaries of ultrafinegrained (UFG) metals produced by severe plastic deformation; therefore, the emission of EGBDs from the grain boundaries could afect the mechanical characteristics of UFG metals. In this paper, we use molecular dynamics to simulate the emission of EGBDs from grain boundaries, and we examine the grain boundary structure dependence of the emissions. Then we apply $J$-integral analysis to evaluate the Peach-Koehler force required for the grain boundaries to emit the EGBD. It can be confirmed that the Peach-Koehler force required to emit the EGBD is highly dependent on the relationship between the Burgers vector components of the EGBD and intrinsic grain boundary dislocations (IGBDs), which form the equilibrium grain boundaries. Comparing analyses of the linear elastic theory with atomic simulations, we confirm that nonlinear structural changes in the dislocation cores of the EGBD and IGBDs, which can only be expressed by atomic scale resolution, are responsible for such strong grain boundary structural dependence. We also verify that normal stress components perpendicular to the slip plane of EGBDs have a significant effect on the emission of EGBDs. [doi:10.2320/matertrans.MD201124]

(Received August 1, 2011; Accepted September 22, 2011; Published December 25, 2011)

Keywords: grain boundary, dislocation, dislocation source, ultrafine-grained metal, molecular dynamics, Peach-Koehler force, J-integral

\section{Introduction}

Recently, several researchers have investigated severe plastic deformation ${ }^{1-3)}$ method for the bulk production of ultrafine-grained (UFG) metals having submicrometer grain sizes; the mechanical properties of UFG metals differ from those of coarse-grained metals having grain sizes in the supermicrometer range. ${ }^{4-6)}$ In general, annealing at temperatures below recrystallization point softens coarse-grained metals and decreases their dislocation density. Recently, it has been reported that the annealing of UFG metals not only decreases their dislocation density but also increases their yield stress, ${ }^{4)}$ as opposed to the case of coarse grained metals. This anomalous phenomenon could occurs when the dislocation sources in the grains become depleted. ${ }^{4)}$ Asdeformed UFG metals by cold rolling after the annealing process decreases their yield stress and improves their ductility, while the grain sizes remain unchanged. ${ }^{4)}$ Such a variation in mechanical characteristics is attributed to the micronization of the grain sizes to a few hundred nanometers, which drastically increases the volume ratio of the grain boundaries. Therefore, we can infer that the mechanical characteristics of the grain boundaries afects the mechanical characteristics of UFG metals.

In general, the equilibrium grain boundary is composed of the intrinsic grain boundary dislocation (IGBD), which is necessary to accommodate misorientations between adjacent grains. According to recent reports, grain boundaries in UFG metals obtained via severe plastic deformation contain the geometrically unnecessary extrinsic grain boundary dislocations (EGBDs), in addition to IGBDs. ${ }^{7,8)}$ Thus, under an applied load, EGBDs are expected to be released from the grain boundaries before the emission of IGBDs if the Burgers vectors of the EGBDs are in close proximity to the Burgers vectors of the lattice dislocations. ${ }^{9)}$ Accordingly, on the basis

*Graduate Student, Kanazawa University of the hypothesis that the ability of the grain boundaries to act as dislocation sources affects the mechanical characteristics of UFG metals, the relationship between the annealing process and these mechanical characteristics can be analyzed as follows. As-deformed UFG metals contain high levels of EGBDs in their grain boundaries; however, when UFG metals are annealed, the EGBDs decompose and change into IGBDs, i.e., non-equilibrium grain boundaries change into equilibrium grain boundaries. This implies that as-deformed UFG metals are capable of emitting EGBDs, in contrast to annealed UFG metals. It has been reported that the yield stress of UFG metals differs under compressive deformation and tensile deformation. ${ }^{10)}$ This tension/compression asymmetry can be explained by the pressure dependence of the dislocation self-energy during bow-out from a grain boundary dislocation source. ${ }^{10)}$ Consequently, we can infer that the ability of grain boundaries to act as dislocation sources has a significant impact on the mechanical characteristics of UFG metals in which a large number of dislocation sources are not easily produce in the grains.

In this study, we employ molecular dynamics (MD) to examine the effects of the relationship between the components of the Burgers vectors of IGBDs and EGBDs in the grain boundaries on the emission of EGBDs from the grain boundaries. For equilibrium grain boundaries with different grain boundary structures, we specifically introduce lattice dislocations externally, and we develop a nonequilibrium grain boundary model containing EGBDs. Then, we apply loads to the models in various directions. In order to evaluate $g$ the dependence of the EGBD emissions on the grain boundary structure, we calculate the Peach-Koehler (PK) force when the EGBDs are released into the grains. We perform $J$-integral analysis around the EGBDs to calculate the PK force in the slip direction because the $J$-integral is equivalent to the PK force. ${ }^{11)}$ The method for calculating the $J$-integral in atomic and finite element models has been proposed previously. ${ }^{12,13)}$ The influence of the normal stress 
components perpendicular to the slip plane on the EGBD emissions is also investigated in order to consider the tension/compression asymmetry of the dislocation source activity in the grain boundaries. Finally, we discuss the effect of the nonlinear structural changes in the dislocation cores of the EGBD and IGBDs on the emission phenomena by comparing the results of atomic simulations (which indicate structural changes in the grain boundary dislocation cores) with the linear elastic theory (which only expresses the elastic interactions of the IGBDs and EGBDs).

\section{Analysis Model and Analysis Conditions}

\subsection{Analysis model}

Figure 1(a) shows an overview of the analysis model. The analyzed element is aluminum, and its properties are expressed by the embedded atom method proposed by Mishin et al. ${ }^{14)}$ The analysis model is based on a bicrystal in which grain I and grain II are periodically placed along the $z$-axis so that the grain boundaries are parallel to the $x$-axis. We set the basic crystal orientations in the [1힐 direction along the $x$-axis, [ $\overline{1} \overline{1} 2]$ direction along the $y$-axis, and [ $\overline{1} \overline{1} \overline{1}]$ direction along the $z$-axis. Then, we express the crystal orientations for grain I and grain II by rotating the basic crystal orientations in the clockwise direction about the $y$-axis by $\pm \theta$, respectively; hence, the grain boundaries used in this study are the $\langle 112\rangle$ tilt grain boundaries. In this study, we use symmetrical tilt grain boundaries of $\Sigma 21$ and $\Sigma 15$. For the $\Sigma 21$ boundary, $\theta$ is set at $67.8^{\circ}$, and for $\Sigma 15, \theta$ is set at $50.8^{\circ}$. In this study, we refer to $\Sigma 21$ as model $A$ and $\Sigma 15$ as model B. The grain boundary misorientation angles of $\Sigma 21$ and $\Sigma 15$ are $\theta_{\Sigma 21}=44.42^{\circ}$ and $\theta_{\Sigma 15}=78.46^{\circ}$, respectively. $\Sigma 21$ and $\Sigma 15$ each consists of two types of structural units. ${ }^{15-17)}$ The two types of structural units that constitute the $\Sigma 21$ boundary are called $A$ and $B$ structural units. Under this classification, the grain boundary structure of $\Sigma 21$ has a structural unit periodicity of $|B B A|$. On the other hand, the two types of structural units that constitute the $\Sigma 15$ boundary are called $B$ and $C$ structural units (the $B$ structural unit is identical to that for $\Sigma 21$ ); thus, the $\Sigma 15$ boundary has a structural unit periodicity of $|B B C|$. Using an energetically stable $\Sigma 11$ boundary with $\theta_{\Sigma 11}=62.96^{\circ}$ as a reference structure, IGBDs in the $A$ and $C$ structural units are indicated in gray in Figs. 1(b) and 1(c). ${ }^{17)}$ The $A$ structural unit contains the IGBD with the Burgers vector $\boldsymbol{b}_{\Sigma 21}^{\mathrm{igb}}=2 a / 11[3 \overline{1} 1]$, which possesses the $z$ orientation component only, whereas the $C$ structural unit contains the IGBD wih $\boldsymbol{b}_{\Sigma 15}^{\text {igb }}=-2 a$ / $11[3 \overline{1} 1]$, whose direction is opposite to that of $\boldsymbol{b}_{\Sigma 21}^{\mathrm{igb}}$. In this study, $a$ denotes the lattice constant, i.e. $a=0.405 \mathrm{~nm}$.

Under an applied load, a set of lattice dislocations is introduced, as indicated in black in Fig. 1(a), into the grain boundaries from the grain I, and a non-equilibrium grain boundary containing EGBDs is created, as indicated in white in Fig. 1(a). The upper-left and lower-right regions where lattice dislocations are impinged are called $S_{1}$ and $S_{2}$, respectively. As shown in Figs. 1(b) and 1(c), the structural units where the EGBD is introduced at $\mathrm{S}_{1}$ and $\mathrm{S}_{2}$ are not identical. Subsequently, we perform structure relaxation of non-equilibrium grain boundaries under no applied load using the conjugate gradient method, and then, we obtain stable
Table 1 Applied strain directions and inner products of Burgers vectors of IGBDs and EGBD.

\begin{tabular}{ccccc}
\hline grain boundary & \multicolumn{2}{c}{$\Sigma 21$} & \multicolumn{2}{c}{$\Sigma 15$} \\
\hline model & $\mathrm{A}_{1}$ & $\mathrm{~A}_{2}$ & $\mathrm{~B}_{1}$ & $\mathrm{~B}_{2}$ \\
\hline $\boldsymbol{b}^{\text {igb }} \cdot \boldsymbol{b}^{\text {egb }}$ & + & - & - & + \\
\hline load direc. & z-com. $x$-ten. $z$-ten. $x$-com. & $z$-com. $x$-ten. $z$-ten. $x$-com. \\
\hline
\end{tabular}

non-equilibrium grain boundary models that contains EGBDs. Depending on the EGBD's Burgers vector component introduced, the models in this study are referred to as model $\mathrm{A}_{i}$ and $\mathrm{B}_{i}$; thus, when $i=1$, EGBDs are introduced from grain I via tensile preloading in the $z$ direction, as shown in Fig. 2(a), and when $i=2$, EGBDs are introduced from grain I via compressive preloading in the $z$-direction, as shown in Fig. 2(b). Therefore, the Burgers vectors of the introduced EGBDs are set to $\boldsymbol{b}_{\Sigma 21}^{\text {egb }}$ and $-\boldsymbol{b}_{\Sigma 21}^{\text {egb }}$ for model $A_{1}$ and model $A_{2}$, respectively, and $\boldsymbol{b}_{\Sigma 15}^{\mathrm{egb}}$ and $-\boldsymbol{b}_{\Sigma 15}^{\mathrm{egb}}$ for model $\mathrm{B}_{1}$ and model $\mathrm{B}_{2}$, respectively. Here, $\boldsymbol{b}_{\Sigma 21}^{\text {egb } 15}=a / 2[110]$, which is equivalent to the lattice edge dislocation. Therefore, we confirm that for models $\mathrm{A}_{1}$ and $\mathrm{B}_{2}, \boldsymbol{b}^{\text {igb }} \cdot \boldsymbol{b}^{\text {egb }}>0$, whereas for models $\mathrm{A}_{2}$ and $\mathrm{B}_{1}, \boldsymbol{b}^{\text {igb }} \cdot \boldsymbol{b}^{\text {egb }}<0$. This indicates the difference in the interactions of IGBDs and EGBDs for each model, which can be understood from the non-equilibrium grain boundary structure for each model, as shown in Fig. 7.

\subsection{Analysis conditions}

For EGBD emission to the grain I area, we perform deformation tests in the $x$ and $z$ directions on each model in order to direct the PK force on the EGBD toward grain I. Table 1 lists the deformation direction for each model. We apply the periodic boundary condition in all directions and we set the strain rate at $\varepsilon_{x}, \varepsilon_{z}= \pm 2 \times 10^{8} 1 / \mathrm{s}$. The unit cell length in the $y$-direction is fixed, whereas the other unit cell length is controlled to maintain the normal stress at zero. ${ }^{18)}$ The analysis temperature is maintained at $0.1 \mathrm{~K}$.

A comparison of models $A_{1}$ and $A_{2}\left(B_{1}\right.$ and $\left.B_{2}\right)$, which have different signs of the inner product $\boldsymbol{b}^{\text {igb }} \cdot \boldsymbol{b}^{\text {egb }}$, enables us to investigate the effect of the Burgers vector components of IGBDs and EGBDs on the emission of EGBDs. Moreover, comparing the results of different loading directions facilitates a better understanding of the effect of the normal stress component which is perpendicular to the slip plane of the EGBD shown in Fig. 2, the emission of EGBDs.

\section{PK Force Evaluation Method and Its Validity}

In this study, we use $J$-integral analysis ${ }^{19)}$ to calculate the PK force acting on EGBDs. In the following subsections, we provide an overview of the $J$-integral calculation method, and we verify its validity for an atomic model. ${ }^{12)}$

\subsection{J-Integral analysis of atomic models}

Nakatani et al. ${ }^{12)}$ proposed a method for calculating the $J$-integral value for an MD simulation. When we calculate the PK force acting on the EGBD in the $x_{1}$ slip direction, $f_{x_{1}}$, shown in Fig. 1(a), using the $J$-integral value, the $J$-integral value can be expressed by the following equation in the $\mathrm{O}-x_{1} x_{2} x_{3}$ coordinates in Fig. 1(a). 


$$
\begin{aligned}
J=f_{x_{1}}= & \sum_{\alpha \text { in } \mathrm{S}^{*}}\left[\left(W^{C(\alpha)}-W^{O(\alpha)}\right) \delta_{1 j}\right. \\
& \left.-\left(\sigma_{i j}^{C(\alpha)}-\sigma_{i j}^{O(\alpha)}\right) \frac{\partial u_{i}^{(\alpha)}}{\partial x_{1}}\right] \frac{\partial Q}{\partial x_{j}} V^{(\alpha)} \frac{1}{l_{2}}
\end{aligned}
$$

Here, $\mathrm{S}^{*}$ denotes the area represented by $\mathrm{S}^{*}$ in Fig. 1(a), and $W^{(\alpha)}, \sigma_{i j}^{(\alpha)}$, and $u_{i}^{(\alpha)}$ denote the potential energy, stress component, and displacement component of the $\alpha$ atom in $\mathrm{O}-x_{1} x_{2} x_{3}$, respectively. The $l_{2}$ denotes the length in the $y$-direction in the analysis model, whereas $V^{(\alpha)}$ denote the occupied volume of the $\alpha$ atom; we simply use the atomic volume of the perfect crystal instead of $V^{(\alpha)}$ in this study. The letters $O$ and $C$ denote a non-loading state and deformation state, respectively. $Q$ is a weighting function introduced to express the line integral performed by the original $J$-integral analysis in the form of the surface integral for the $\mathrm{S}^{*}$ region in Fig. 1(a). Moreover, $Q$ is a continuous function that becomes 0 at the internal diameter $r_{1}$ and 1 at the outer diameter $r_{2} . r$ denotes the distance from the dislocations. In this study, $Q(r)$ is defined as

$$
Q(r)=\frac{1}{2}\left\{1-\cos \pi \frac{r-r_{1}}{r_{2}-r_{1}}\right\} .
$$

The displacement gradient $\partial u_{i}^{(\alpha)} / \partial x_{1}$ can be obtained as

$$
\left(\frac{\partial u_{i}}{\partial x_{1}}\right)^{(\alpha)}=-\sum_{\beta=1}^{N} u^{(\beta)} \frac{\partial w}{\partial x_{1}} V^{(\beta)} .
$$

Here, the $\beta$ atom is located within $0.6 \mathrm{~nm}$ of the $\alpha$ atom. $w$ is a three-dimensional normal distribution with the $\alpha$ atom as the original point, and the distribution value refers to the distance of the first neighbor atoms $\left(r_{1 \mathrm{st}}=0.286 \mathrm{~nm}\right)$, which is set as $r_{1 \mathrm{st}}^{2}$. When a homogeneous strain $\varepsilon_{x}^{\text {app }}$ was applied to the perfect crystal atomic model, we confirmed that the strain of the $\alpha$ atom, $\varepsilon_{x}^{\mathrm{MD}}=\partial u_{x}(\alpha) / \partial x$, evaluated by eq. (3), was the same as $\varepsilon_{x}^{\text {app }}$.

\subsection{Evaluation of PK force acting on Lomer sessile dislocation}

In order to verify the validity of the PK force calculated using eq. (1), we calculate the $J$-integral values of a straight lattice dislocation under shear deformation. In this study, aluminum is used as the analysis metal; it induces smaller Peierls stresses for lattice edge dislocations. Therefore, lattice edge dislocations can move easily if a shear strain is applied. In order to constrain the movement of dislocations for calculating the $J$-integral values under applied stress, we adopt the Lomer sessile dislocation with a much higher Peierls stress.

As shown in Fig. 3(a), we developed a square shape atomic model (side, $120 \mathrm{~nm}$ ), with the Lomer sessile dislocation at the center. Then, we relaxed the analysis model under a free surface condition in the $x$ and $z$ directions by using the conjugate gradient method. The relaxed atomic configuration is shifted according to the homogeneous shear strain $\gamma_{z x}^{\text {app }}$, and then, all regions, except for the central part at a radius of $25 \mathrm{~nm}$ are removed. The surrounding black region of the cylinder region shown in Fig. 3(b) is fixed during the relaxation of the interior containing the Lomer sessile dislocation. Using this atomic structure, PK forces acting on the Lomer sessile dislocation are calculated by the following two methods. The PK forces obtained by the two methods are compared in order to investigate the validity of eq. (1) for the estimation of the PK force in atomic models.

In the first method, the PK force is calculated from the average shear stress $\bar{\tau}_{z x}^{\mathrm{MD}}$ applied to the cylinder region, as shown in Fig. 3(b). Because the relation between the shear stress $\tau_{z x}$ and the PK force $f_{x}$ is given by $f_{x}=\tau_{z x} b$ when the direction of the Burgers vector of the dislocation is parallel to the $x$-direction, as shown in Fig. 3(b), substituting $\bar{\tau}_{z x}^{\mathrm{MD}}$ for $\tau_{z x}$ provides the PK force. Here, $b$ denotes the Burgers vector for the Lomer sessile dislocation. This method is referred to as method $1\left(\bar{\tau}_{z x}^{\mathrm{MD}}\right)$. In the second method, called method 2 ( $J$-integral), the PK force is obtained using eq. (1). In this method, the internal diameter $r_{1}$ is fixed at $5 \mathrm{~nm}$, and the outer diameter $r_{2}$ is changed from $6 \mathrm{~nm}$ to $12 \mathrm{~nm}$ in order to evaluate the dependence of the integral areas $\mathrm{S}^{*}$ on the $J$-integral values, as shown in Fig. 4(b).

Figure 4(a) shows PK forces obtained by the two methods. A comparison of the results of method 1 and method 2 confirms a linear increase in PK forces in proportion to an increase in shear strains for both methods. Thus, it is possible to use eq. (1) to evaluate the PK force in atomic models. The PK force obtained by method 2 is slightly larger than that obtained by method 1 owing to the brought by the boundary condition, which constrains the atomic displacement in the black regions shown in Fig. 3(b). Next, we examine the dependence of the outer diameter $r_{2}$. Figure 4(c) shows the relationship between the PK force and the outer diameter $r_{2}$ at $0.2 \%$ shear strain. We can confirm that $r_{2}$ is slightly dependent on the PK force when it is set at $9 \mathrm{~nm}$ or more. Therefore, in this study, we set $r_{1}=5 \mathrm{~nm}$ and $r_{2}=10 \mathrm{~nm}$ to evaluate the PK force acting on the EGBD.

\subsection{Effects of normal stress on PK force}

In this section, we investigate the change in the PK force obtained by eq. (1) when $\varepsilon_{z}$ is applied to the analysis model in shear deformation. The dislocation theory argues that vertical loads on the slip plane of the dislocation have no impact on the glide movements of the edge dislocations.

We evaluate the PK force acting on the Lomer sessile dislocation by applying both shear strain $\gamma_{z x}$ and normal strain $\varepsilon_{z}= \pm 0.1$ and \pm 0.4 on the previously mentioned Lomer sessile dislocation model, and we perform $J$-integral analysis around the dislocations. Figure 5 shows the dependence of the PK force on the normal strain $\varepsilon_{z}$ under shear strain $\gamma_{z x}$. Figure 5 shows no significant difference in each $\varepsilon_{z}$ under $\gamma_{z x}$; hence, the $J$-integral value also shows a smaller impact of the normal strain on the PK force evaluated by eq. (1) as well as the dislocation theory.

\subsection{J-Integral that crosses the grain boundaries}

The $\mathrm{S}^{*}$ for the $J$-integral calculation contains the grain boundaries, as shown in Fig. 1(a); therefore, the elastic anisotropy may affects the obtained PK force. In order to examine this effect, first we apply a strain of $\varepsilon_{z}=2 \%$ to model $A$ of $\Sigma 21$ with equilibrium grain boundaries and model $A_{1}$ with non-equilibrium grain boundaries. Then, we apply $J$-integral analysis to these models for various PK force directions. The results are shown in Fig. 6. It can be 


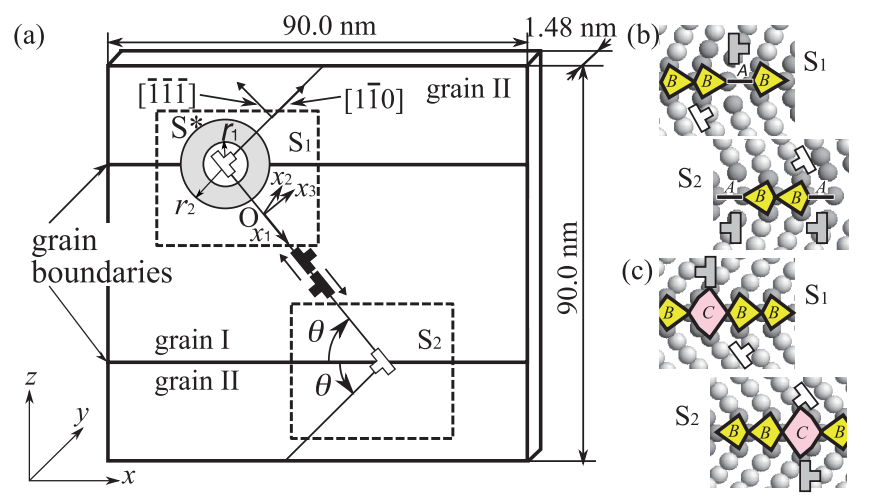

Fig. 1 (a) Schematic figure of the analysis model. (b) Structural units of $\Sigma 21$ (model A). (c) Structural units of $\Sigma 15$ (model B).

(a) $\quad$ model $\mathrm{A}_{1}, \mathrm{~B}_{1}$

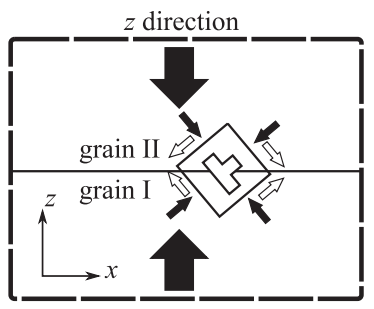

$x$ direction

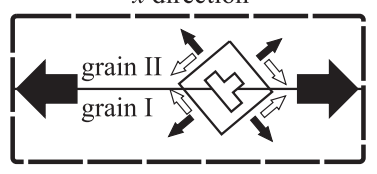

Fig. 2 Applied load directions and stress components for the EGBD in $\mathrm{S}_{1}$ region. The big black arrows show the applied load directions. The small white and black arrows show the stress components of the normal and shear stresses acting on the EGBDs.

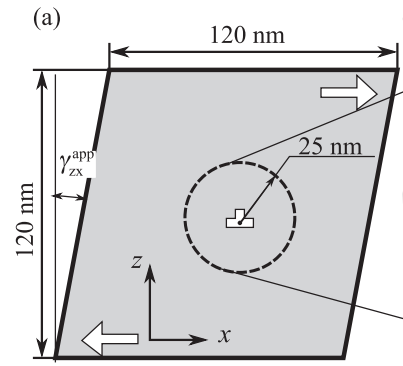

(b)

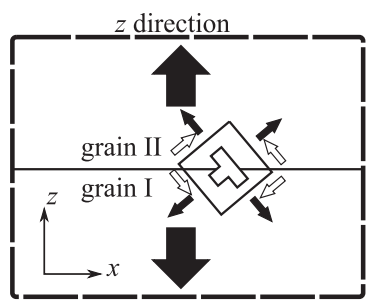

$x$ direction
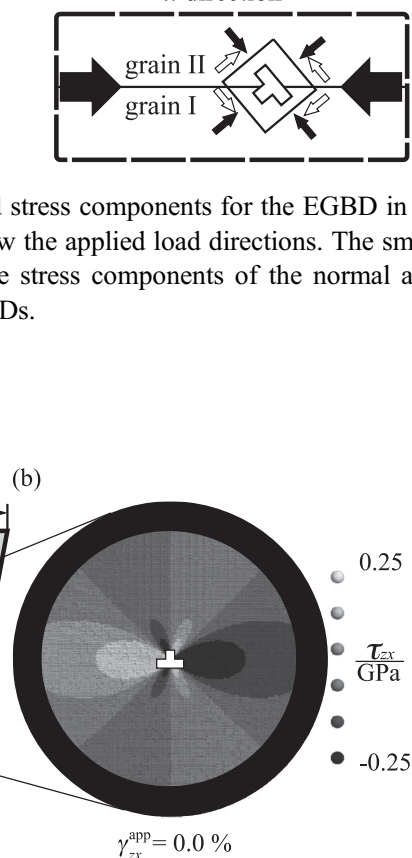

Fig. 3 Lomer sessile dislocation model.

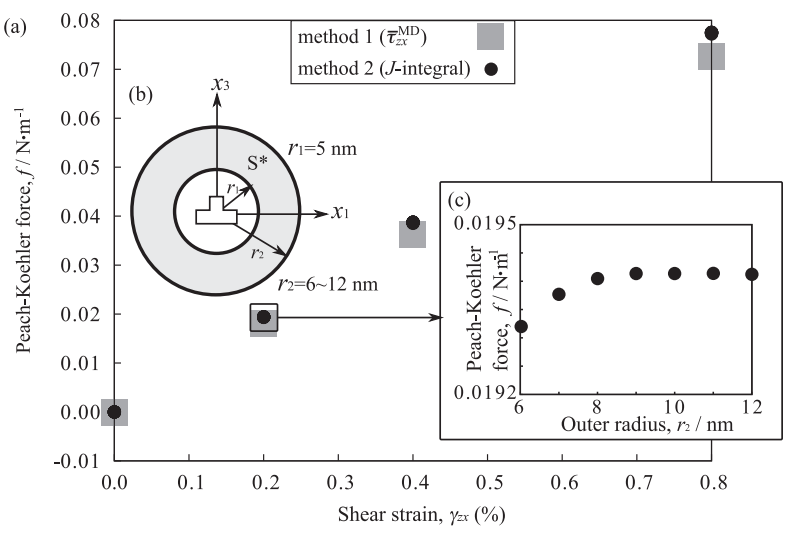

Fig. 4 (a) PK force vs. shear strain. (b) Integral region for $J$ integral analysis. (c) Outer cutoff $r_{2}$ dependence of $J$-integral.

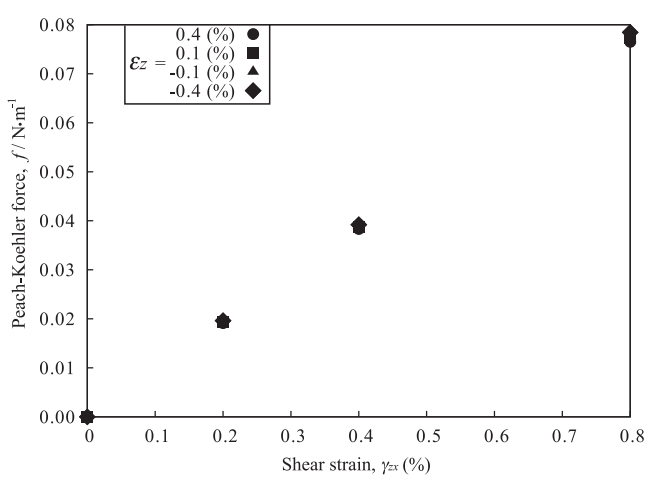

Fig. 5 Normal strain $\varepsilon_{z}$ dependence of the Peach-Koehler force under shear strain.

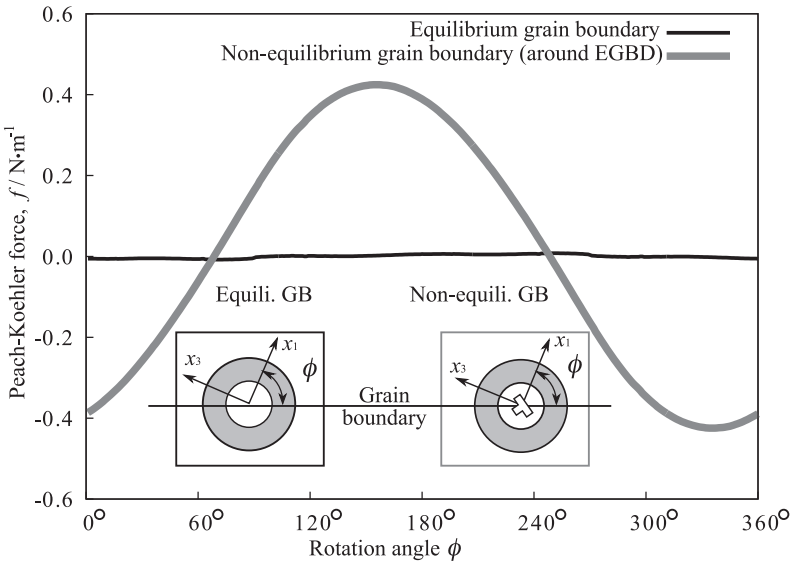

Fig. $6 J$-integral values containing equilibrium and non-equilibrium grain boundaries. (a) model $\mathrm{A}_{i}$

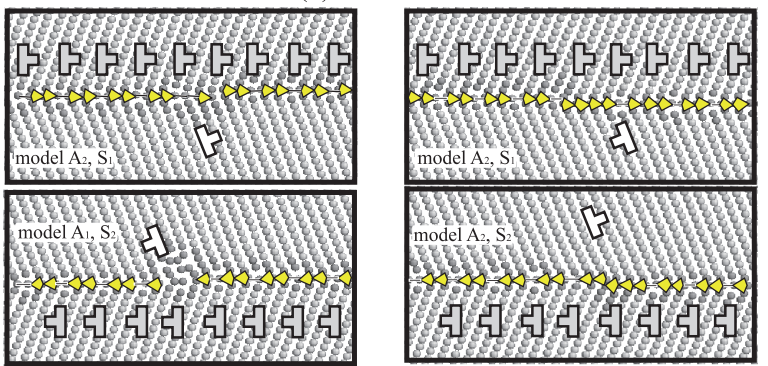

(b) model B

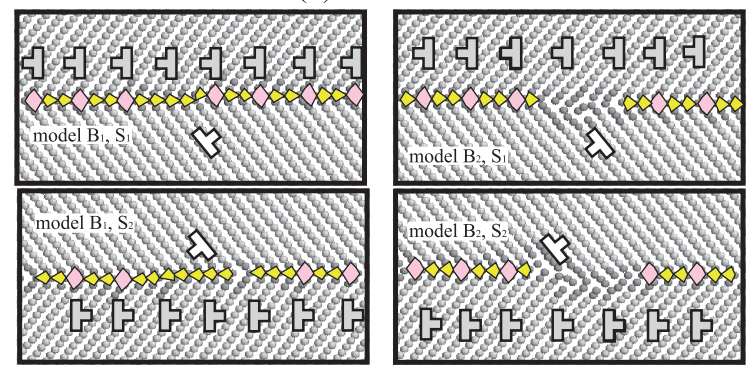

Fig. 7 Non-equilibrium grain boundary structures of each analysis model. 
confirmed that the PK force acting on the EGBD, indicated in gray, fluctuates periodically, and that the PK force becomes maximum/minimum when the $x_{1}$ directions that express the direction of the PK force action and the direction of the EGBD Burgers vectors are identical. On the other hand, the PK force becomes almost zero for the equilibrium grain boundaries with no EGBD. Thus, strong elastic anisotropy is not generated. This could be due to the fact that the anisotropic factor obtained from the potential energy employed in this study is 1.25 , which is closer to 1 . The grain boundary slightly affects the $J$-integral analysis, and hence, $J$-integral analysis can be adopted to evaluate the EGBD pinning effect across grain boundaries, when calculating the PK force acting on the EGBD in aluminum.

\section{Analysis Results}

\subsection{Non-equilibrium grain boundary structures}

Figure 7 shows the non-equilibrium grain boundary structures near the EGBD. When $\boldsymbol{b}^{\text {igb }} \cdot \boldsymbol{b}^{\text {egb }}>0$ in models $A_{1}$ and $B_{2}$, the grain boundary structures near the EGBD impinged are in disarray; hence, it is not possible to express the grain boundary structures in the structural unit. On the other hand, when $\boldsymbol{b}^{\text {igb }} \cdot \boldsymbol{b}^{\text {egb }}<0$, which makes the residual Burgers vector after the reaction of $\boldsymbol{b}^{\text {igb }}$ and $\boldsymbol{b}^{\text {egb }}$ much smaller than that when $\boldsymbol{b}^{\text {igb }} \cdot \boldsymbol{b}^{\text {egb }}>0$, the grain boundary structures with the EGBD in model $\mathrm{A}_{2}$ and model $\mathrm{B}_{1}$ are less disorganized than those of models $\mathrm{A}_{1}$ and $\mathrm{B}_{2}$. Hence, it is possible to express the structures in the structural unit. This result shows that the non-equilibrium grain boundary structures that contain the EGBD are highly dependent on the relationship between the Burgers vector components of the IGBDs and EGBDs.

In order to examine the relationship between non-equilibrium grain boundary structures and stress fields, the distributions of the first invariant of stress $I_{1}$ of models $\mathrm{B}_{1}$ and $B_{2}$ are shown in Fig. 8. Figure 8 shows similarities in the size of the stress fields near the EGBD for both grain boundary structures in spite of the differences shown in Fig. 7. This means that the Burgers vectors of the lattice dislocations impinged at the grain boundaries are preserved in the local areas of the grain boundaries. It also implies that the EGBD stress field affects the mechanical characteristics in the grain, and we can speculate that this effect is not sensitive to the non-equilibrium grain boundary structures.

\subsection{Extrinsic grain boundary dislocation emissions from grain boundaries}

We perform deformation simulations with the conditions listed in Table 1 for EGBD emission into grain I from the grain boundaries. Figures 9 and 10 show the stress-strain curves obtained from models $\mathrm{A}_{1} / \mathrm{A}_{2}$ and $\mathrm{B}_{1} / \mathrm{B}_{2}$, respectively. The vertical and horizontal axes represent the normal stress and strain along the loading directions, respectively. The upper horizontal axis represents the strain when the models are deformed in the $x$-direction, whereas the lower axis represents the strain when the models are deformed in $z$-direction. It is important to note that the strains in the $x$-direction and $z$-direction have opposite signs. The solid circles represent the first EGBD emissions and the numbers indicate the values of the strains at the time of the EGBD emissions. Both Figures also show the sites where EGBDs were emitted. Table 2 lists the critical applied stresses $\sigma_{\mathrm{c}}$ at the time of the EGBD emissions as well as the sites where the EGBDs were emitted. To compare the abilities of the nonequilibrium grain boundaries to act as dislocation sources, we perform deformation tests on the equilibrium grain boundaries with no EGBD; the stress when the first dislocation occurs is stated in parentheses in Table 2. The simulations show that all non-equilibrium grain boundaries experience dislocation emission with lower stresses, as compared the equilibrium grain boundaries without the EGBD. This implies that it is easier to emit EGBDs than to induce dislocations from the equilibrium grain boundaries, and that non-equilibrium grain boundaries are more capable of acting as dislocation sources than equilibrium grain boundaries.

Figure 9 (Fig. 10) shows that model $\mathrm{A}_{1}$ (model $\mathrm{B}_{2}$ ) with $\boldsymbol{b}^{\text {igb }} \cdot \boldsymbol{b}^{\text {egb }}>0$ emits the EGBD with lower stresses than model $\mathrm{A}_{2}\left(\right.$ model $\left.\mathrm{B}_{1}\right)$ with $\boldsymbol{b}^{\text {igb }} \cdot \boldsymbol{b}^{\text {egb }}<0$. When $\boldsymbol{b}^{\text {igb }} \cdot \boldsymbol{b}^{\text {egb }}>0$ for models $\mathrm{A}_{1}$ and $\mathrm{B}_{2}$, the introduced EGBD was emitted directly from the sites; in other words, the slip planes of the impinged lattice dislocation at the grain boundaries and the emitted dislocation from the grain boundaries are the same. However, when $\boldsymbol{b}^{\text {igb }} \cdot \boldsymbol{b}^{\text {egb }}<0$ for models $A_{2}$ and $B_{1}$, a different dislocation is emitted from the grain boundaries near the site where the EGBD exists. In other words, as Fig. 7 suggests, the structurally disorganized models $\mathrm{A}_{1}$ and $\mathrm{B}_{2}$ tend to emit the EGBD easily, whereas it is difficult for models $A_{2}$ and $B_{1}$, whose grain boundary structures can be re-expressed by the structural unit. This shows that the structural change in dislocation cores by the interactions of IGBDs and EGBD has a strong impact on the emission of EGBDs.

Next, we investigate the relationship between the EGBD pinning effects of the grain boundaries and the normal stress components perpendicular to the slip planes. Figure 2 shows that the normal stress components perpendicular to the slip planes of the EGBD become positive when tensile loads are applied in the $x$ - or $z$-directions. On the other hand, the normal stress components become negative when compressive loads are applied in the $x$ - or $z$-directions. In order to examine the influence of normal stress components on the emission of EGBDs, we compare $\left|\sigma_{\mathrm{c}}^{c} / \sigma_{\mathrm{c}}^{t}\right|$ for each model. Here, $\sigma_{\mathrm{c}}^{c}$ and $\sigma_{\mathrm{c}}^{t}$ represent the critical stress for the first EGBD emission under compressive and tensile loading, respectively. Model $\mathrm{A}_{1}\left(\boldsymbol{b}^{\mathrm{igb}} \cdot \boldsymbol{b}^{\mathrm{egb}}>0\right)$ has $\left|\sigma_{\mathrm{c}, \mathrm{A}_{1}}^{z c} / \sigma_{\mathrm{c}, \mathrm{A}_{1}}^{x t}\right|=1.38$, and model $\mathrm{A}_{2} \quad\left(\boldsymbol{b}^{\mathrm{igb}} \cdot \boldsymbol{b}^{\text {egb }}<0\right)$ has $\left|\sigma_{\mathrm{c}, \mathrm{A}_{2}}^{x c} / \sigma_{\mathrm{c}, \mathrm{A}_{2}}^{z A_{1}}\right|=1.74$. Model $\mathrm{B}_{1}\left(\boldsymbol{b}^{\text {igb }} \cdot \boldsymbol{b}^{\text {egb }}<0\right)$ has $\left|\sigma_{\mathrm{c}, \mathrm{B}_{1}}^{z c} / \sigma_{\mathrm{c}, \mathrm{B}_{1}}\right|=1.58$, and model $\mathrm{B}_{2}\left(\boldsymbol{b}^{\mathrm{igb}} \cdot \boldsymbol{b}^{\text {egb }}>0\right)$ has $\left|\sigma_{\mathrm{c}, \mathrm{B}_{2}}^{x c} / \sigma_{\mathrm{c}, \mathrm{B}_{2}}^{z t}\right|=1.12$. It can be confirmed that it is more difficult to emit EGBDs from grain boundaries under the normal compressive stress component perpendicular to the slip plane, as compared to the case of the normal tensile stress component; therefore, the influence of normal stress components on the emission of EGBDs could cause tensile/compressive asymmetry in UFG metals. ${ }^{10)}$ The obtained results imply that the non-equilibrium grain boundaries with $\boldsymbol{b}^{\text {igb }} \cdot \boldsymbol{b}^{\text {egb }}<0$, whose grain boundary structures can be re-expressed by the structural units, are more sensitive to normal stress components perpendicular to the slip planes. 
Table 2 Critical applied stresses $\left(\sigma_{\mathrm{c}}\right)$ and critical Peach-Koehler forces acting on EGBD $\left(f_{\mathrm{c}}\right)$ for the emission of the EGBD from grain boundaries for each model in the atomic simulations. Values in parentheses denote critical applied stresses for emitting dislocations from equilibrium grain boundaries without any EBGD. $f_{\mathrm{c}}^{\mathrm{T}}$ denotes critical Peach-Koehler forces acting on EGBD, estimated by the linear elastic thoery.

\begin{tabular}{|c|c|c|c|c|c|c|c|c|}
\hline grain boundary & \multicolumn{4}{|c|}{$\Sigma 21$} & \multicolumn{4}{|c|}{$\Sigma 15$} \\
\hline model & \multicolumn{2}{|c|}{$\mathrm{A}_{1}$} & \multicolumn{2}{|c|}{$\mathrm{A}_{2}$} & \multicolumn{2}{|c|}{$\mathrm{B}_{1}$} & \multicolumn{2}{|c|}{$\mathrm{B}_{2}$} \\
\hline $\boldsymbol{b}^{\mathrm{igb}} \cdot \boldsymbol{b}^{\mathrm{egb}}$ & \multicolumn{2}{|c|}{+} & \multicolumn{2}{|c|}{-} & \multicolumn{2}{|c|}{-} & \multicolumn{2}{|c|}{+} \\
\hline load direc. & $z$-com. & $x$-ten. & $z$-ten. & $x$-com. & $z$-com. & $x$-ten. & $z$-ten. & $x$-com. \\
\hline$\left(\sigma_{\mathrm{c}}^{\mathrm{eq}} / \mathrm{GPa}\right)$ & $(-5.6)$ & $(4.7)$ & $(7.5)$ & $(-13.6)$ & $(-7.1)$ & $(5.3)$ & $(4.9)$ & $(-6.7)$ \\
\hline dislo. emission & $\mathrm{S}_{1}$ & $\mathrm{~S}_{1}$ & $\mathrm{~S}_{2}$ & $\mathrm{~S}_{2}$ & $\mathrm{~S}_{1}$ & $\mathrm{~S}_{1}$ & $\mathrm{~S}_{1}$ & $\mathrm{~S}_{1}$ \\
\hline$\sigma_{\mathrm{c}} / \mathrm{GPa}$ & -1.8 & 1.3 & 3.9 & -6.8 & -4.1 & 2.6 & 1.9 & -2.2 \\
\hline$\left|\sigma_{\mathrm{c}}^{c} / \sigma_{\mathrm{c}}^{t}\right|$ & \multicolumn{2}{|c|}{$\left|\sigma_{\mathrm{c}, \mathrm{A}_{1}}^{z c} / \sigma_{\mathrm{c}, \mathrm{A}_{1}}^{x t}\right|=1.38$} & \multicolumn{2}{|c|}{$\left|\sigma_{\mathrm{c}, \mathrm{A}_{2}}^{x c} / \sigma_{\mathrm{c}, \mathrm{A}_{2}}^{z t}\right|=1.74$} & \multicolumn{2}{|c|}{$\left|\sigma_{\mathrm{c}, \mathrm{B}_{1}}^{z c} / \sigma_{\mathrm{c}, \mathrm{B}_{1}}^{x t}\right|=1.58$} & \multicolumn{2}{|c|}{$\left|\sigma_{\mathrm{c}, \mathrm{B}_{2}}^{x c} / \sigma_{\mathrm{c}, \mathrm{B}_{2}}^{z t}\right|=1.12$} \\
\hline$f_{\mathrm{c}} / \mathrm{N} \cdot \mathrm{m}^{-1}$ & 0.23 & 0.18 & 0.50 & $0.52^{*}$ & 0.80 & 0.48 & 0.35 & 0.44 \\
\hline$\left|f_{\mathrm{c}}^{c} / f_{\mathrm{c}}^{t}\right|$ & $\left|f_{\mathrm{c}, \mathrm{A}_{1}}^{z c}\right|$ & $=1.28$ & $\left|f_{\mathrm{c}, \mathrm{A}_{2}}^{x c}\right|$ & $=1.04^{*}$ & $\left|f_{\mathrm{c}, \mathrm{B}_{1}}^{z c}\right|$ & $=1.67$ & $\mid f_{\mathrm{c}, \mathrm{B}_{2}}^{x c}$ & $=1.26$ \\
\hline$f_{\mathrm{c}}^{\mathrm{T}} / \mathrm{N} \cdot \mathrm{m}^{-1}$ & \multicolumn{2}{|c|}{0.61} & \multicolumn{2}{|c|}{0.01} & \multicolumn{2}{|c|}{0.08} & \multicolumn{2}{|c|}{0.62} \\
\hline
\end{tabular}

\subsection{Peach-Koehler force acting on the EGBD in atomic simulations}

In previous sections, the emission of dislocations from the grain boundaries was estimated using loading stresses. However, it is not easy to compare the structure dependence of the abilities of various grain boundaries to act as dislocation source with different misorientation angles because of the difference in the Schmid factor of the slip systems for activating EGBDs. Therefore, we evaluate the PK force in the slip direction, which acts on EGBD, using $J$-integral analysis, and we compare the emission ability of EGBDs for $\Sigma 21$ and $\Sigma 15$ grain boundaries.

Figures 11 and 12 show the PK force for emitting the first dislocation from non-equilibrium grain boundaries in models $\mathrm{A}_{i}$ and $\mathrm{B}_{i}$, respectively. The solid circles in Figs. 11 and 12 indicate the timings of the dislocation emissions, whereas the numbers represent the critical $\mathrm{PK}$ force $f_{\mathrm{c}}$ for the first dislocation emissions. These values are listed in Table 2. All PK forces increase linearly until the first dislocation emission, except for the compressive deformation in the $x$-direction for model $\mathrm{A}_{2}$. The reason is shown in Fig. 13, where the distributions of the first invariant of stress $I_{1}$ are subtracted from the average $\bar{I}_{1}$ of the analysis model immediately after the emissions of the EGBD in model $\mathrm{A}_{2}$. In most cases, grain boundary structures within the $\mathrm{S}^{*}$ region for $J$-integral analysis do not change until the first dislocation emission, as shown in Fig. 13(a). However, in the case of the compressive deformation in the $x$-direction for model $\mathrm{A}_{2}$ shown in Fig. 13(b), the grain boundary structure changes within the $\mathrm{S}^{*}$ region before the first dislocation emission; therefore, we cannot obtain the exact PK force by calculating the $J$-integral.

Comparing the PK force confirms that the emission of dislocations from the grain boundaries is highly dependent on $\boldsymbol{b}^{\mathrm{igb}} \cdot \boldsymbol{b}^{\mathrm{egb}}$, as shown in Table 2. Furthermore, we can examine the pinning effects of EGBD for the $\Sigma 21$ and $\Sigma 15$ grain boundaries by comparing the PK force. For models $\mathrm{A}_{1}$ and $\mathrm{B}_{2}\left(\boldsymbol{b}^{\mathrm{igb}} \cdot \boldsymbol{b}^{\mathrm{egb}}>0\right)$, the $\Sigma 15$ grain boundary requires a larger PK force then the $\Sigma 21$ grain boundary to emit the EGBD, owing to the influence of normal stress components on the emission of EGBDs $f_{\mathrm{c}, \mathrm{B}_{2}}^{x c}>f_{\mathrm{c}, \mathrm{A}_{1}}^{z c}$ and $f_{\mathrm{c}, \mathrm{B}_{2}}^{z t}>f_{\mathrm{c}, \mathrm{A}_{1}}^{x t}$. Although $\Sigma 21$ and $\Sigma 15$ are very closely related in terms of the Burgers vectors of IGBDs when $\Sigma 11$ is used as a reference structure, as mentioned in section 2.1, the $\Sigma 21$ boundary has lower EGBD pinning effects than the $\Sigma 15$ boundary. This means that nonlinear structure changes in the cores of IGBDs and EGBDs, which could not be easily estimated by superposing their atomic configurations as described in section 4.1, have a strong impact on the emission of EGBDs.

\subsection{Peach-Koehler force acting on the EGBD in linear elastic theory}

In this section, in order to further investigate the effect of nonlinear structure changes in the cores of IGBDs and EGBDs on the emission of EGBDs, we compare the EGBD pinning effects obtained by expressing the elastic interactions of IGBDs and EGBDs within the framework of the linear elastic theory, with the results of atomic simulations.

On the basis of an energetically stable $\Sigma 11$ as a structural reference, the grain boundary structure of $\Sigma 21$ (model A) and $\Sigma 15$ (model B) in this study can be expressed by placing IGBDs to accommodate the misorientations from $\Sigma 11$. In general, the small misorientations $\theta$ of the grain boundaries can be expressed as ${ }^{20)}$

$$
\sin \frac{\theta}{2}=\frac{b}{2 h}
$$

Here, $h$ and $b$ denote the dislocation interval and Burgers vector of the lattice dislocation, respectively. Using the linear elastic theory, we can express the grain boundary structures of $\Sigma 21$ and $\Sigma 15$ by the superposition of IGBDs.

The misorientation between $\Sigma 21$ and $\Sigma 11, \theta_{\Sigma 21}-\theta_{\Sigma 11}$, is $-18.54^{\circ}$, and the misorientation between $\Sigma 15$ and $\Sigma 11, \theta_{\Sigma 15}-\theta_{\Sigma 11}$, is $15.50^{\circ}$. The IGBD interval $h_{\Sigma 21}$ that 


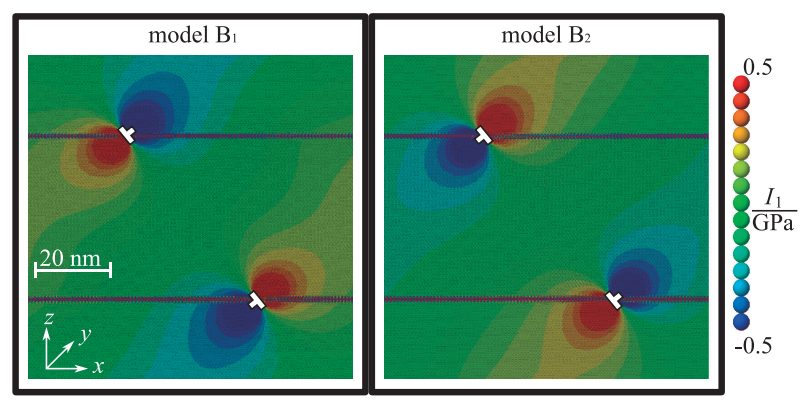

Fig. 8 Stress distributions of the first invariant stress $I_{1}$ of models $\mathrm{B}_{1}$ and $\mathrm{B}_{2}$.

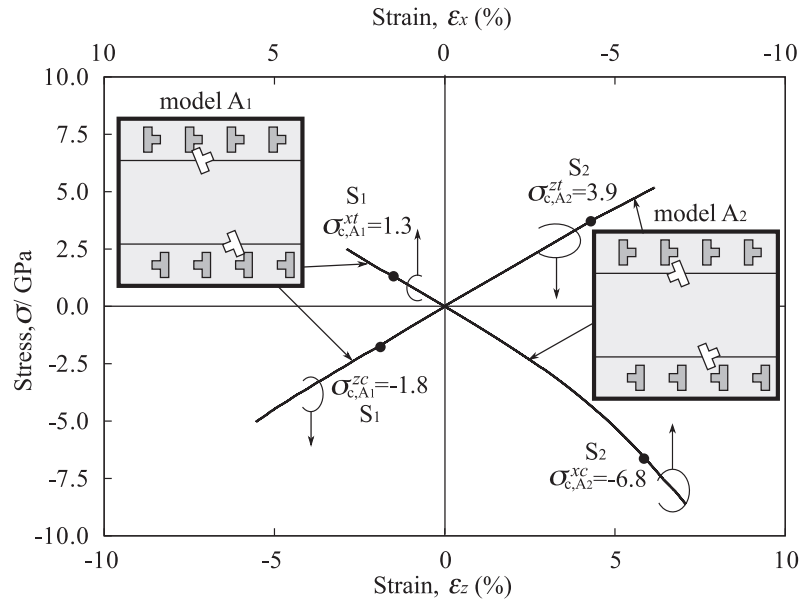

Fig. 9 Stress-strain curves of models $A_{1}$ and $A_{2}$.

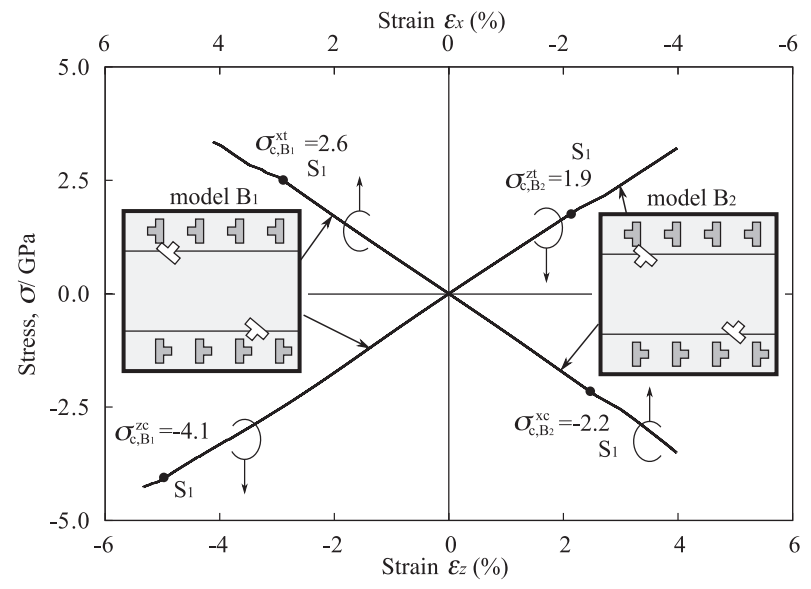

Fig. 10 Stress-strain curves of models $\mathrm{B}_{1}$ and $\mathrm{B}_{2}$.

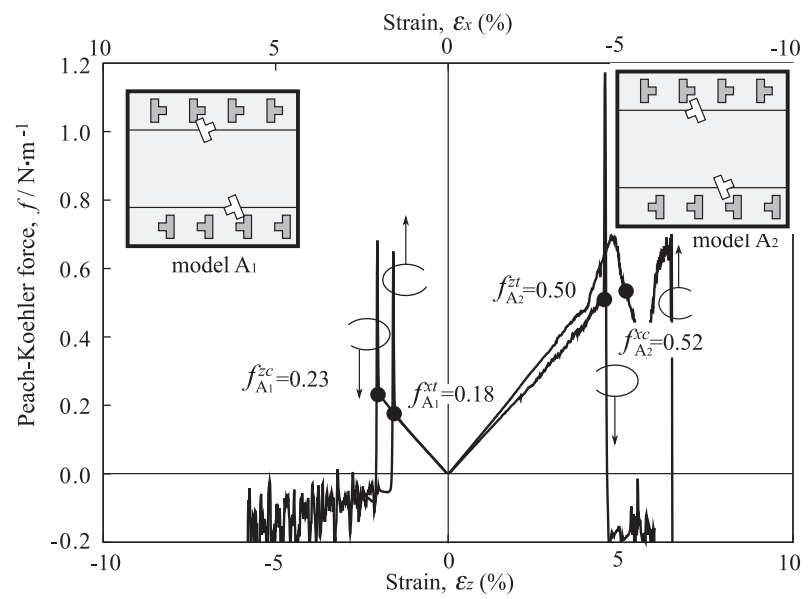

Fig. 11 PK forces estimated by $J$-integral analysis in models $\mathrm{A}_{1}$ and $\mathrm{A}_{2}$.

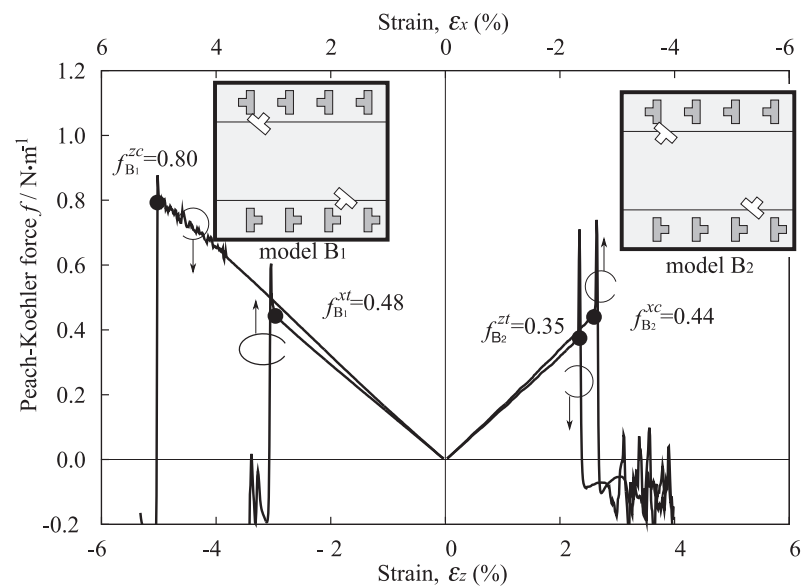

Fig. 12 PK forces estimated by $J$-integral analysis in models $\mathrm{B}_{1}$ and $\mathrm{B}_{2}$.

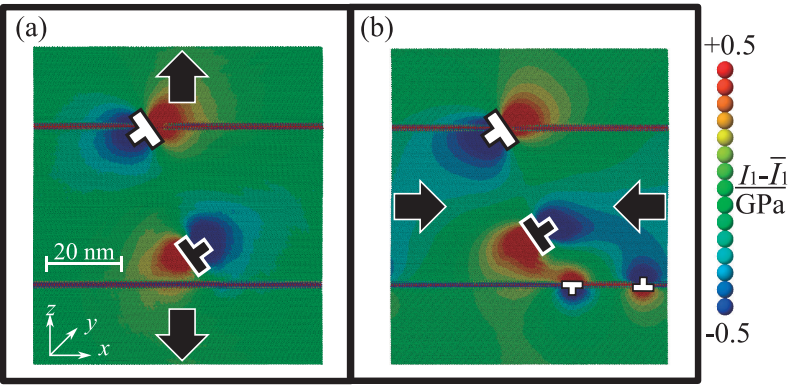

Fig. 13 Stress distributions of the first invariant of stress $I_{1}$ subtracted from the average $\bar{I}_{1}$ of the analysis model when the EGBD is emitted from grain boundaries in model $\mathrm{A}_{2}$ under different load directions.

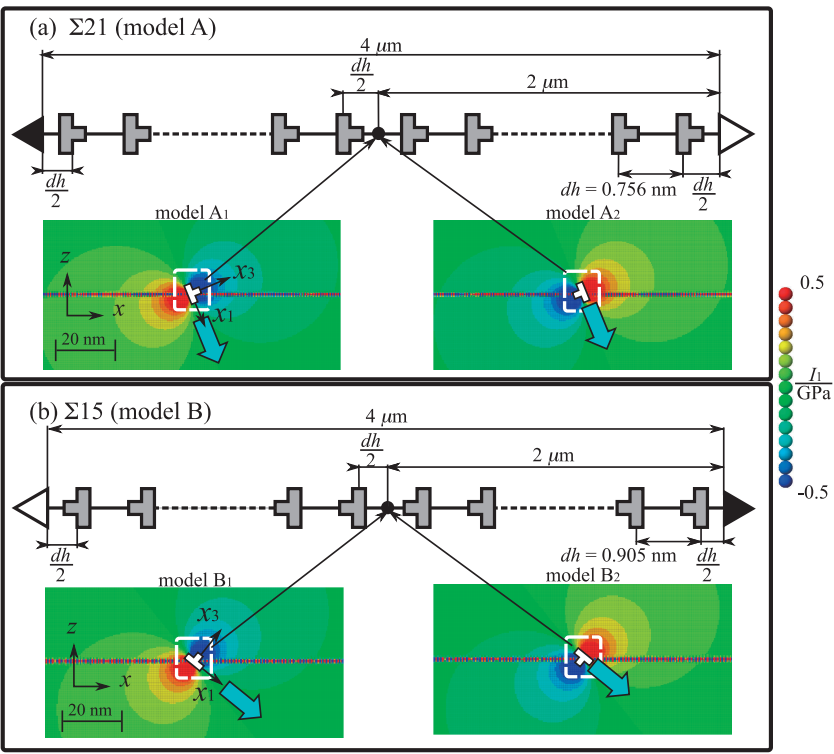

Fig. 14 Grain boundary structures expressed by the combination of the finite IGBD array and the disclination dipole. Stress distributions of first invariant of stress $I_{1}$ of non-equilibrium grain boundaries expressed by the superposition of stress fields of IGBDs, the disclination dipole, and the EGBD using the linear elastic theory. 
expresses the misorientation $\theta_{\Sigma 21}-\theta_{\Sigma 11}$ is $0.756 \mathrm{~nm}$ from eq. (4), whereas the IGBD interval $h_{\Sigma 15}$ that expresses the misorientation $\theta_{\Sigma 15}-\theta_{\Sigma 11}$ is $0.905 \mathrm{~nm}$ because the size of the Burgers vector of IGBDs is $b_{\Sigma 21}^{\text {igb }}=b_{\Sigma 15}^{\text {igb }}=2 a / \sqrt{11}=$ $0.244 \mathrm{~nm}$. It should be noted that the IGBD intervals $h_{\Sigma 21}$ and $h_{\Sigma 15}$ in equilibrium grain boundaries are smaller than $4 b^{\text {igb}}$; hence, each dislocation core region that cannot be treated by the linear elastic theory might overlap with each other when the $\Sigma 21$ and $\Sigma 15$ boundaries are expressed by the superposition of IGBDs under the linear dislocation theory. The length of the finite dislocation array is set to approximately $4 \mu \mathrm{m}\left(5292 h_{\Sigma 21}\right.$ for $\Sigma 21$ and $4420 h_{\Sigma 15}$ for $\Sigma 15$ ), as shown in Fig. 14, and we also locate disclinations using a Frank vector with $\theta_{\Sigma 21}-\theta_{\Sigma 11}$ and $\theta_{\Sigma 15}-\theta_{\Sigma 11}$ on both sides of the dislocation arrays in order to remove the long-range stress fields of finite dislocation arrays. ${ }^{21)}$ For simplicity, an EGBD is placed at the center between the IGBDs located $2 \mu \mathrm{m}$ from the disclination. Figure 14 shows the results obtained by the superposition of the first invariant of the stresses of the IGBDs and the EGBD using the linear elastic theory. This shows a high consistency with the nonequilibrium grain boundaries in the atomic model, shown in Fig. 8, and it can be confirmed that the long-range stress field far from the core regions can be expressed by the superposition of IGBDs with the linear elastic theory, even though each core region might overlap.

Next, we move the EGBD in the direction indicated by the arrows in Fig. 14, and we calculate the PK force acting on the EGBD from the elastic interaction evaluated by the dislocation theory. In Fig. 15, the vertical axis indicates the PK force $f^{\mathrm{T}}$ that acts on the EGBD, and the horizontal axis represents the displacement $d x_{1}$ of the EGBD from the grain boundaries. In models $\mathrm{A}_{1}$ and $\mathrm{B}_{2}$, the force that pulls the EGBD in the direction of the grain boundaries becomes activated when the EGBD exists near the grain boundaries. Therefore, the EGBD stably exists at the grain boundaries. On the other hand, in models $\mathrm{A}_{2}$ and $\mathrm{B}_{1}$, the EGBD receives a repulsive force from the grain boundaries, and then, it takes locations in front of the grain boundaries at a distance of $0.67 \mathrm{~nm}$ for model $B_{1}$ and $0.75 \mathrm{~nm}$ for model $A_{2}$ under no applied load. The solid circles in Fig. 15 represent the critical PK force $f_{\mathrm{c}}^{\mathrm{T}}$ required for EGBD emissions from each

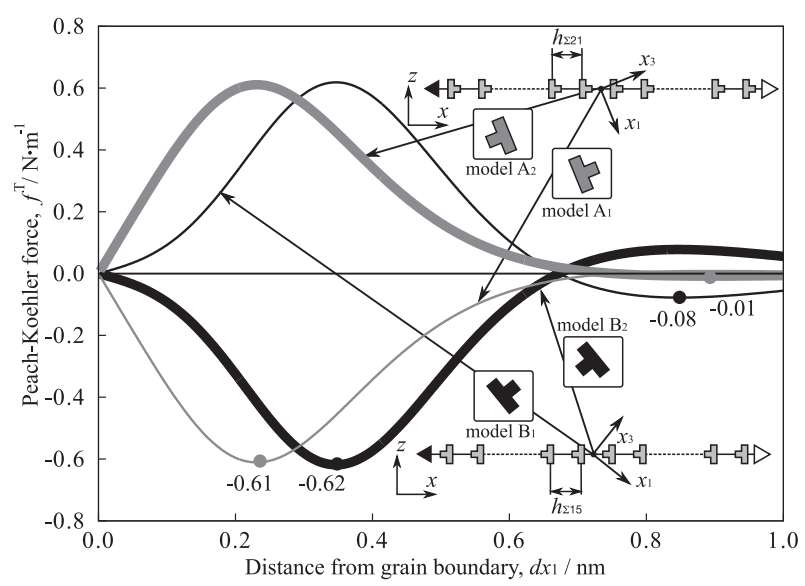

Fig. 15 PK forces acting on EGBDs estimated by the dislocation theory. location, and it is $0.62 \mathrm{~N} / \mathrm{m}$ and $0.61 \mathrm{~N} / \mathrm{m}$ in model $A_{1}$ and model $\mathrm{B}_{2}$, respectively. In model $\mathrm{A}_{2}$ and model $\mathrm{B}_{1}$, it is $0.01 \mathrm{~N} / \mathrm{m}$ and $0.08 \mathrm{~N} / \mathrm{m}$, respectively. These values are listed in Table 2. Therefore, it can be confirmed that $f_{\mathrm{c}}^{\mathrm{T}}$ for $\mathrm{A}_{1}$ and $\mathrm{B}_{2}\left(\boldsymbol{b}^{\text {igb }} \cdot \boldsymbol{b}^{\text {egb }}>0\right)$ is larger than that one for $\mathrm{A}_{2}$ and $\mathrm{B}_{1}$ $\left(\boldsymbol{b}^{\text {igb }} \cdot \boldsymbol{b}^{\text {egb }}<0\right)$.

We compare $f_{\mathrm{c}}$ obtained from MD simulations with $f_{\mathrm{c}}^{\mathrm{T}}$ obtained by the superposition of the stress fields with the linear elastic theory. The results obtained from MD simulations show a small critical PK force and low EGBD pinning effects for $\mathrm{A}_{1}$ and $\mathrm{B}_{2}\left(\boldsymbol{b}^{\mathrm{igb}} \cdot \boldsymbol{b}^{\text {egb }}>0\right)$. However, for $\mathrm{A}_{2}$ and $\mathrm{B}_{1}\left(\boldsymbol{b}^{\mathrm{igb}} \cdot \boldsymbol{b}^{\text {egb }}<0\right)$, the EGBD pinning effects, which are obtained from the elastic interaction with the linear elastic theory, are opposite to the MD results, and the PK force is extremely small. In addition, a comparison between $\Sigma 21$ and $\Sigma 15$ shows no difference in the EGBD pinning effects estimated by the linear elastic theory, indicating the difference between them and the results obtained from MD simulations. The result enables us to understand that in addition to the elastic interaction affecting the EGBD pinning effects, the nonlinear structure changes in the grain boundary dislocation cores, expressed by the atomic simulations shown in Fig. 7, also affect the pinning effects. In other words, it is difficult to evaluate the EGBD pinning effects simply from the superposition of the stress fields of IGBDs and the EGBD.

\section{Conclusions}

In this study, we developed non-equilibrium grain boundary models that contain the EGBD, and we examined the EGBD pinning effects of the symmetrical tilt grain boundaries using atomic simulations. We obtained the following results regarding on the EGBD pinning effects of the grain boundaries.

(1) The relationship between the Burgers vector components of the IGBDs and EGBDs has a strong impact on the structures of non-equilibrium grain boundaries.

(2) The non-equilibrium grain boundaries are capable of emitting the EGBD with lower stress than that required to induce the dislocations from the equilibrium grain boundaries. Thus, non-equilibrium grain boundaries have greater abilities to act as dislocation sources than equilibrium grain boundaries.

(3) The nonlinear structure changes in the dislocation cores of the IGBDs and the EBGD have a strong impact on the emission of EGBDs. Moreover, the study shows that the normal stress components perpendicular to the EGBD slip planes affect the emission of EGBDs.

(4) Performing $J$-integral analysis around the EGBD makes it possible to calculate the $\mathrm{PK}$ force required to emit the EGBD, and it indicates the pinning effects of the EGBD for different grain boundaries.

(5) Evaluating the elastic interaction of IGBDs and the EGBD with the linear elastic theory and comparing this with the results of the atomic models enables us to understand that it is important to consider the structure changes in the dislocation cores from atomic-scale perspectives in order to analyze the emission of EGBDs. 


\section{Acknowledgment}

This study was financially supported by the a Grant-inAid for Scientific Research on Innovative Area, Bulk Nanostructured Metals, through MEXT, Japan (contract No. 22102006 and 22102007), and the support is gratefully appreciated.

\section{REFERENCES}

1) N. Tsuji, Y. Saito, S. H. Lee and Y. Minamino: Adv. Eng. Mater. 5 (2003) 338-344.

2) Y. Saito, H. Utsunomiya and N. Tsuji: Acta Mater. 47 (1999) 579-583.

3) Z. Horita, K. Matsubara and K. Makii: Scr. Mater. 47 (2002) 255-260.

4) X. Huang, N. Hansen and N. Tsuji: Science 312 (2006) 249-251.

5) N. Tsuji, Y. Ito and Y. Saito: Scr. Mater. 47 (2002) 893-899.

6) M. Tanaka, K. Higashida, T. Shimokawa and T. Morikawa: Mater. Trans. 50 (2009) 56-63.

7) Z. Horita, D. Smith, M. Furukawa, Z. Ruslan and G. Terence: J. Mater. Res. 11 (1996) 1880-1890.
8) J. Wang, Y. Iwahashi, Z. Horita and M. Furukawa: Acta Mater. 44 (1996) 2973-2982.

9) D. Hull and D. J. Bacon: Introduction to dislocations, (ButterworthHeinemann, Oxford, 2001) p. 155.

10) S. Cheng and J. Spencer: Acta Mater. 51 (2003) 4505-4518.

11) J. Weertman: Dislocation based fracture mechanics, (World Scientific Publishing Co. Pte. LTD., 1996) pp. 57-58.

12) K. Nakatani, A. Nakatani, Y. Sugiyama and H. Kitagawa: AIAA J. 38 (2000) 695-701.

13) R. Gracie, J. Oswald and T. Belytschko: J. Mech. Phys. Solids 56 (2008) 200-214.

14) Y. Mishin, M. Mehl, D. Papaconstantopoulos, A. Voter and J. Kress: Phys. Rev. B 63 (2001) 224106.

15) A. Sutton and V. Vitek: Phil. Trans. R. Soc. Lond. A 309 (1983) 1-36.

16) R. Balluffi and P. Bristowe: Surf. Sci. 144 (1984) 28-43.

17) T. Shimokawa: Phys. Rev. B 82 (2010) 174122.

18) M. Parrinello and A. Rahman: Phys. Rev. Lett. 45 (1980) 1196-1199.

19) J. R. Rice: J. Appl. Mech. 35 (1968) 379-386.

20) J. Hirth and J. Lothe: Theory of dislocations, (John Wiley and Sons, New York, 1982) p. 705.

21) S. Bobylev, M. Gutkin and I. Ovidko: Acta Mater. 52 (2004) 37933805 . 